\title{
DETECÇÃO DE FALHAS EM MOTORES ELÉTRICOS ATRAVÉS DA CLASSIFICAÇÃO DE PADRÕES DE VIBRAÇÃO UTILIZANDO UMA REDE NEURAL ELM
}

\author{
G. L. B. RAMALHO, A. H. PEREIRA, P. P. REBOUÇAS FILHO e C. M. S. MEDEIROS \\ Instituto Federal do Ceará \\ gramalho@ifce.edu.br
}

Artigo submetido em janeiro/2014 e aceito em maio/2014

DOI: $10.15628 /$ holos.2014.1925

\section{RESUMO}

O monitoramento das condições dos motores elétricos industriais fornece informações que auxiliam o planejamento das intervenções de manutenção antes da ocorrência de falhas. Este trabalho propõe uma nova abordagem para o monitoramento de condições operacionais de motores de indução trifásicos baseada na extração de características de um sinal de vibração obtido com acelerômetros MEMS. Os dados extraídos da decomposição do sinal de vibração, por Transformada Haar e através da dimensão fractal, são utilizadas para treinar uma rede neural ELM. Através do resultado de experimentos é demonstrada a viabilidade da metodologia para aplicação na detecção e identificação de falhas mecânicas e elétricas.

PALAVRAS-CHAVE: Detecção de falhas, sensores MEMS, rede neural ELM.

\section{FAULT DETECTION IN INDUCTION MOTORS USING VIBRATION PATTERNS AND ELM NEURAL NETWORK}

\begin{abstract}
The condition monitoring of industrial electric motors provides information to help planning maintenance interventions before the occurrence of failures. This paper proposes a new approach for monitoring the operational condition of three-phase induction motors based on the extraction of characteristics of a signal obtained with MEMS accelerometers. The data extracted
\end{abstract}

from the decomposition of the vibration signal using Haar Transform and the fractal dimension are used to train a ELM neural network. The results of our experiments demonstrated the feasibility of the proposed methodology in detection and identification of mechanical and electrical failures..

KEYWORDS: Fault detection, MEMS sensors, ELM neural network. 


\section{INTRODUÇÃO}

O desempenho de uma máquina, ao longo de sua vida útil, pode ser rapidamente degradado a partir do surgimento dos primeiros sinais de falha. Quando as condições operacionais da máquina começam a mudar, o primeiro efeito é o aumento ou mudança do padrão de vibração seguida por uma perda de desempenho. Se nenhuma ação for tomada, em geral decorrente do não monitoramento do equipamento, ocorre o aumento de ruído audível e uma redução significativa de desempenho. Antes que a vibração atinja níveis excessivos uma interrupção na operação para dar lugar a procedimentos de manutenção pode reduzir a extensão dos danos e prolongar a vida útil do equipamento.

Existem diferentes métodos para monitoramento de equipamentos industriais em modo periódico ou contínuo (on line). Alguns desses métodos utilizam a análise de parâmetros como nível e viscosidade de lubrificantes, a temperatura, ruído e vibração (Nepomuceno, 1989).

Particularmente no caso de análise de vibração, sensores MEMS (Microelectromechanical System) têm sido aplicados no monitoramento de ventiladores (Chen et al., 2010) e de sistemas de transmissão de aerogeradores. No trabalho de Maruthi e Vittal (2005) foram utilizados acelerômetros MEMS em substituição aos tradicionais acelerômetros piezelétricos no monitoramento de falhas em motores monofásicos do tipo gaiola de esquilo. Os autores realizaram análise espectral de falhas a partir do sinal do acelerômetro MEMS e através da assinatura de corrente do motor. No trabalho de Hassan et al. (2013), os autores investigam diferentes aplicações nas quais são empregadas redes neurais tanto no controle quanto no monitoramento de sistemas de potência. Paiva, Galvão e Rodrigues (2009) utilizaram a Transformada wavelet (TW) na filtragem do sinal com o objetivo de detecção de falhas em sistemas dinâmicos. No trabalho de Jiang e Liu (2011), foi investigado o monitoramento de condição de máquina através da extração de características que indicam a deterioração do equipamento. Li et al. (2010) e Li et al. (2011) obtiveram êxito na detecção de falhas em caixas de engrenagens utilizando wavelet.

Máquinas de aprendizagem e redes neurais têm sido bastante exploradas nesse tipo de aplicação. Konar e Chattopadhway (2011) empregaram o uso de extração de características do sinal de vibração baseado em transformada wavelet. A partir das decomposições wavelet foram extraídas medidas e posteriormente estas medidas foram classificadas utilizando uma máquina de vetor de suporte (SVM, do inglês support vector machine). Chakraborty, De e Chakrabarti (2012) propuseram um método de análise de falhas baseado em mapas de características autoorganizáveis (SOM, do inglês auto-organizing maps) e funções de base radial (RBF, do inglês, radial basis function).

Neste artigo é apresentado um método de predição de falhas incipientes com base na extração de características de um sinal de vibração obtido com acelerômetros MEMS. A extração de características emprega decomposições por transformada wavelet Haar e dimensão fractal (Ramalho et al.,2012). Estas características são utilizadas para treinar uma máquina de aprendizagem extrema (ELM).

Os resultados obtidos em ensaios, mostram que defeitos de rolamento, folgas de acoplamento ou curto circuito entre bobinas podem ser detectados e caracterizados com a metodologia proposta. 
Este trabalho está organizado como segue. Na seção 2 é apresentada a metodologia proposta para o monitoramento e identificação de falhas a partir da extração de características do sinal de vibração obtidas com as decomposições da transformada Haar e dimensão fractal. $\mathrm{Na}$ seção 3 é apresentado uma breve descrição dos ensaios. Os resultados e discussões são apresentados na seção 4. As conclusões e sugestões de trabalhos futuros são apresentados na seção 5 .

\section{DETEÇÃO DE FALHAS}

A metodologia proposta, ilustrada na Figura 1, baseia-se na classificação de descritores do grau de complexidade do sinal de vibração de um equipamento. Os descritores são obtidos a partir de sinais de vibração coletados com acelerômetros MEMS, conforme proposto por Ramalho et al. (2012). Utiliza-se o cálculo da dimensão fractal, além da energia e entropia dos primeiros níveis de decomposição wavelet do sinal.

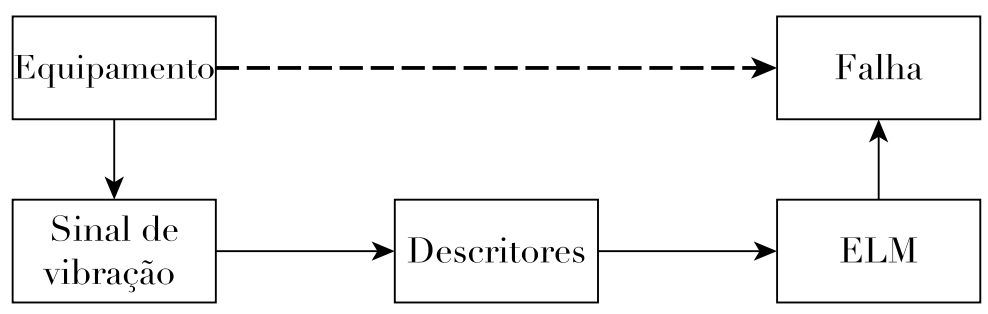

Figura 1 - Metodologia para detecção da falha.

A metodologia utilizada para monitoramento da condição de máquina é baseada na aquisição periódica do sinal de vibração. As características extraídas desse sinal são avaliadas por uma rede neural previamente treinada para condições de falha conhecidas. Quando uma possível falha é detectada é emitido um alarme para acionamento da equipe de manutenção. As falhas são detectadas em seu estágio inicial, antes que causem danos ao equipamento monitorado.

\subsection{Decomposição do sinal de vibração}

Conforme proposto por Ramalho et al. (2012), uma transformada wavelet discreta é utilizada para decompor o sinal de vibração em níveis de detalhes visando a extração dos atributos do sinal. Seja $x$ um sinal unidimensional periódico discreto que representa o sinal de vibração obtido com um acelerômetro MEMS a uma frequência de amostragem $f_{s}$ durante um período $T$ . Extraem-se os coeficientes de aproximação e de detalhes do primeiro nível de decomposição de um sinal discreto $x$ utilizando a Transformada wavelet Haar discreta (Mallat, 1999). Esses coeficientes são definidos, respectivamente, por

$$
\begin{aligned}
& a_{1}[i]=(x[2(i \quad 1)+1]+x[2 i]) / 2 \\
& \text { e } \\
& \text { equação (1) } \\
& d_{1}[i]=x[2(i \quad 1)+1] \quad x[2 i] \\
& \text { equação (2) }
\end{aligned}
$$

em que, $i=1,2, \ldots N / 2$, sendo $N$ o número de amostras do sinal. As decomposições seguintes utilizam as equações (1) e (2), calculadas sobre a componente de aproximação do nível anterior. 


\subsection{Extração de características}

Um sinal de vibração apresenta um padrão estocástico bastante complexo, que pode ser descrito por medidas extraídas das decomposições wavelet Haar do sinal (Ramalho et al.,2012; Ramalho et al.,2013). Supõe-se que estes atributos podem descrever a condição de operação do equipamento monitorado. A partir dessa ideia, Ramalho et al. (2012) definiram algumas medidas para análise do sinal por meio de descritores, a saber: energia, entropia e dimensão fractal.

A energia é obtida a partir dos coeficientes de detalhes $d_{j}$ do sinal. Esse atributo fornece uma estimativa indireta da intensidade de vibração através do nível de variação desses coeficientes. $\mathrm{O}$ atributo de energia é obtido por

$$
E n=\frac{1}{D}{ }_{i=1}^{D} d_{j}[i]^{2},
$$

equação (3)

em que $D$ é a dimensão de $d_{j}$.

A medida da entropia do primeiro nível de detalhes $d_{1}$ aproxima o grau de desorganização do sinal de vibração. Essa medida é sensível à presença de harmônicas, sendo obtida a partir da densidade de probabilidade $p$ do conjunto de coeficientes $d_{1}$, por meio da expressão

$$
E t=p \log p\left(d_{1}\right)
$$

equação (4)

A dimensão fractal é uma métrica que estima o comprimento do desdobramento de um sinal utilizando o algoritmo de contagem de caixas (box counting) (Falconer, 2003) por meio da expressão

$$
D F=\log N_{b} / \log F
$$

em que $N_{b}$ é o número de caixas (boxes) -dimensionais de tamanho $R$ necessárias para cobrir os elementos não nulos do sinal. Para o caso particular de um sinal unidimensional (i.e., $=1$ ), as caixas são representadas por segmentos de reta nele contidos.

As medidas extraídas das decomposições do sinal de vibração formam um vetor de atributos de 3 dimensões $\mathbf{a}=\left\{a_{1}, a_{2}, a_{3}\right\}$, em que $a_{1}=E n, a_{2}=E t$ e $a_{3}=D F$. Esse vetor de atributos constitui um descritor do padrão de vibração para uma determinada condição do motor.

\subsection{Classificação das falhas}

Neste trabalho é empregada uma máquina de aprendizagem extrema (ELM, do inglês Extreme Learning Machine) (Huang, Zhu e Siew, 2004) utilizada na classificação da condição de máquina, visando a detecção de falhas. A ELM é uma rede perceptron multicamada (MLP) que utiliza uma regra de aprendizagem realizada em um único passo, da entrada para a saída (Huang, Zhu e Siew, 2004; Huang, Zhu e Siew, 2006; Wang, Cao e Yuan, 2011). Por isso a sua característica principal é a rapidez de treinamento. A regra geral de aprendizagem dos neurônios de uma ELM é definida por 


$$
\sum_{i=1}^{H} \beta_{i} g\left(\mathbf{w}_{i} \mathbf{x}_{j}+b_{i}\right)=\mathbf{t}_{j} ; j=1, \cdots, N \text {, }
$$

equação (6)

em que $H$ é a quantidade de neurônios da camada oculta e $N$ é o número de vetores de treinamento. Os vetores $\mathbf{w}_{i}=\left[w_{i 1}, w_{i 2}, \cdots, w_{i n}\right]^{\top}$ são os pesos da camada oculta e $\beta_{i}=\left[\beta_{i 1}, \beta_{i 2}, \cdots, \beta_{i m}\right]^{\top}$ são os pesos da camada de saída. No treinamento, para cada entrada $\mathbf{x}_{j}$ de dimensão $n$, apresenta-se o valor conhecido $\mathbf{t}_{j}$ de dimensão $m$. Uma função de ativação diferenciável $g(\rtimes$, em geral do tipo logística, é escolhida para regular assintoticamente a saída da rede.

$$
\begin{gathered}
\text { Considere as matrizes } \mathbf{H}=\left[\begin{array}{ccc}
g\left(\mathbf{w}_{1} \mathbf{x}_{1}+b_{1}\right) & \cdots & g\left(\mathbf{w}_{H} \mathbf{x}_{1}+b_{H}\right) \\
\vdots & \ddots & \vdots \\
g\left(\mathbf{w}_{1} \mathbf{x}_{N}+b_{1}\right) & \cdots & g\left(\mathbf{w}_{H} \mathbf{x}_{N}+b_{H}\right)
\end{array}\right], \beta=\left[\begin{array}{c}
\beta_{1}^{\top} \\
\vdots \\
\beta_{H}^{\top}
\end{array}\right] \\
\text { e } \\
\mathbf{T}=\left[\begin{array}{c}
\mathbf{t}_{1}^{\top} \\
\vdots \\
\mathbf{t}_{N}^{\top}
\end{array}\right] .
\end{gathered}
$$

A regra de aprendizagem definida pela equação (6) pode ser reescrita na forma matricial como $\mathbf{H}=\mathbf{T}$. A solução $=\mathbf{H}^{\dagger} \mathbf{T}$ pode ser obtida pela aplicação da técnica linear dos mínimos quadrados, sendo $\mathbf{H}^{\dagger}$ a pseudo inversa generalizada de Moore-Penrose (Huang, Zhu e Siew, 2004). É possível provar que a rede ELM independe dos pesos da camada oculta $\mathbf{w}_{i}$, os quais podem ser inicializados com valores aleatórios (Huang, Zhu e Siew, 2006).

Após o treinamento da rede ELM, são apresentados dados desconhecidos para obter uma predição da classe à qual esses dados mais se assemelham.

\section{MODELO EXPERIMENTAL}

A medição da vibração foi realizada com um acelerômetro MEMS triaxial. O sensor possui resposta de frequência na faixa $400 \mathrm{~Hz}$, faixa de medição $\pm 1,5 g( \pm 1,6 \mathrm{~V})$, sensibilidade transversal (cross axis) de $\pm 5 \%$ e sensibilidade de $100 \mathrm{mV} / \mathrm{g}$, adequados para os níveis de intensidade e de frequência de vibração monitorados. A frequência de ressonância do sensor é da ordem de $6 \mathrm{kHz}$, bem abaixo da faixa de frequências medidas.

Um acelerômetro MEMS foi fixado à carcaça do motor sobre o mancal dianteiro do eixo. Nessa posição, a medição do sinal de vibração é realizada no sentido axial do eixo do motor. O sinal de vibração foi obtido com um módulo de aquisição de dados de 16 bits. Utilizou-se uma taxa de amostragem de $10 \mathrm{kHz}$ e todas as medições foram realizadas com o motor em regime permanente.

Os experimentos foram conduzidos em uma bancada de ensaios (Oliveira et al., 2013) composta por um motor de indução trifásico com rotor em gaiola, de 1,0 cv, quatro polos, $220 \mathrm{Vca}$ acionado por um conversor de frequência apropriado. Para imposição de carga mecânica, é 
utilizado um freio eletrodinâmico ajustável. A Figura 2 ilustra a bancada de ensaios utilizada nos experimentos, com destaque para posição de fixação do acelerômetro MEMS.

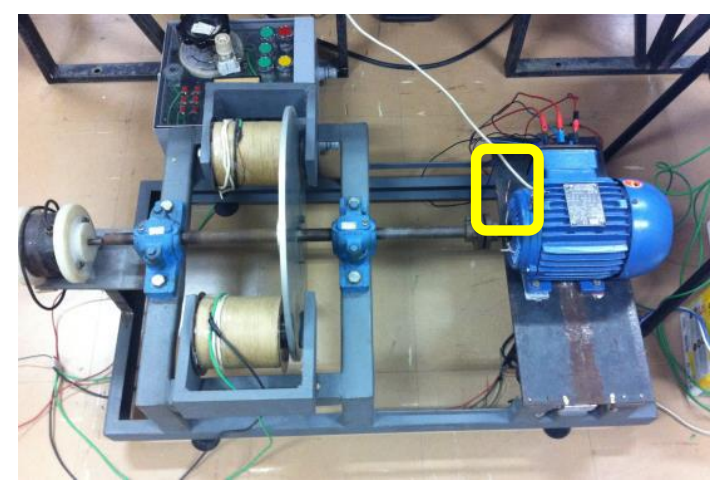

Figura 2 - Equipamento utilizado nos ensaios, compreendendo o freio eletrodinâmico ajustável (à esquerda) e motor de indução (à direita). No detalhe, a indicação do local de fixação do acelerômetro.

\subsection{Aquisição de dados}

O conjunto de dados base para o treinamento dos classificadores contempla, além das condições operacionais normais, diversas combinações de condições operacionais do motor sob falha conforme procedimento descrito por Oliveira et al. (2013).

Os ensaios realizados contemplaram, separadamente, três falhas elétricas causadas por curto circuito estatórico. Para cada falha elétrica, foi utilizado um conjunto de espiras $n_{c}$ adjacentes em uma mesma fase. Esses conjuntos, indicados pelas classes C1, C2 e C3, correspondem às proporções $1,4 \%, 4,9 \%$ e $9,2 \%$ do total de espiras $n_{e}$ de uma mesma fase, respectivamente.

Foram adotadas duas intensidades de curto circuito para cada percentual, denominadas de alta (a) e baixa impedância (b), como indicado na Tabela 1. A intensidade do curto circuito é definida pela utilização de uma impedância em paralelo com uma dado percentual de espiras. A operação sem falha, denominada operação normal $(\mathrm{N})$, é adotada como referência. Para cada condição de operação foi empregada uma carga mecânica constante, com níveis de 0\%, 50\% e $100 \%$ em relação à corrente nominal do motor. A coleta do sinal de vibração foi realizada em cada nível de carga mecânica para frequências distintas de alimentação, com intervalo de $5 \mathrm{~Hz}$, compreendidas entre $30 \mathrm{~Hz}$ e $60 \mathrm{~Hz}$.

Uma rotina de medição da condição de máquina corresponde a 10 amostragens consecutivas do sinal de vibração. Uma amostragem compreende 1000 amostras do sinal. Para cada uma das 10 amostragens é calculado um vetor de atributos a. O vetor característico da condição monitorada é obtido tomando-se o valor médio à desses 10 vetores de atributos. 
Tabela 1 - Condições de operação.

\begin{tabular}{c|c|c|c|c}
\hline $\begin{array}{c}\text { Relação de espiras } \\
\text { em curto }\end{array}$ & \multicolumn{4}{|c}{ Classes } \\
\cline { 2 - 5 } & $\mathrm{C} 1$ & $\mathrm{C} 2$ & $\mathrm{C} 3$ & $\mathrm{~N}$ \\
\hline $\mathrm{n}_{\mathrm{c}}$ & 5 & 17 & 32 & 0 \\
\hline $\mathrm{n}_{\mathrm{c}} / \mathrm{n}_{\mathrm{e}}$ & $1,4 \%$ & $4,9 \%$ & $9,2 \%$ & - \\
\hline intensidade & $\mathrm{a}, \mathrm{b}$ & $\mathrm{a}, \mathrm{b}$ & $\mathrm{a}, \mathrm{b}$ & - \\
\hline
\end{tabular}

Fonte - Adaptado de Oliveira et al. (2013).

\section{RESULTADOS E DISCUSSÃO}

\subsection{Extração de atributos}

Três atributos formam o vetor de características da condição de operação do equipamento. Para a extração desses atributos são empregadas as equações (1) e (2) no cálculo de 7 níveis de decomposições wavelet Haar sobre o sinal de vibração. Os valores de energia (En) são obtidos com a equação (3), aplicadas sobre os coeficientes de detalhes das decomposições wavelet do sinal de vibração. A entropia (Et) do sinal, obtida com a equação (4), é calculada sobre o primeiro nível de detalhes da decomposição wavelet. O cálculo da dimensão fractal (DF), obtido com a equação (5), é realizado sobre o sinal de vibração. A Figura 3 ilustra os vetores de atributos médios $\overline{\mathbf{a}}$ para as condições de operação normal $(\mathrm{N})$ e para três condições de falha $(\mathrm{C} 1, \mathrm{C} 2, \mathrm{C} 3)$, obtidas em 3 níveis de carga (0\%,50\% e 100\%) nas frequências de acionamento de $30 \mathrm{~Hz}$ até $60 \mathrm{~Hz}$ em intervalos de 5 $\mathrm{Hz}$.

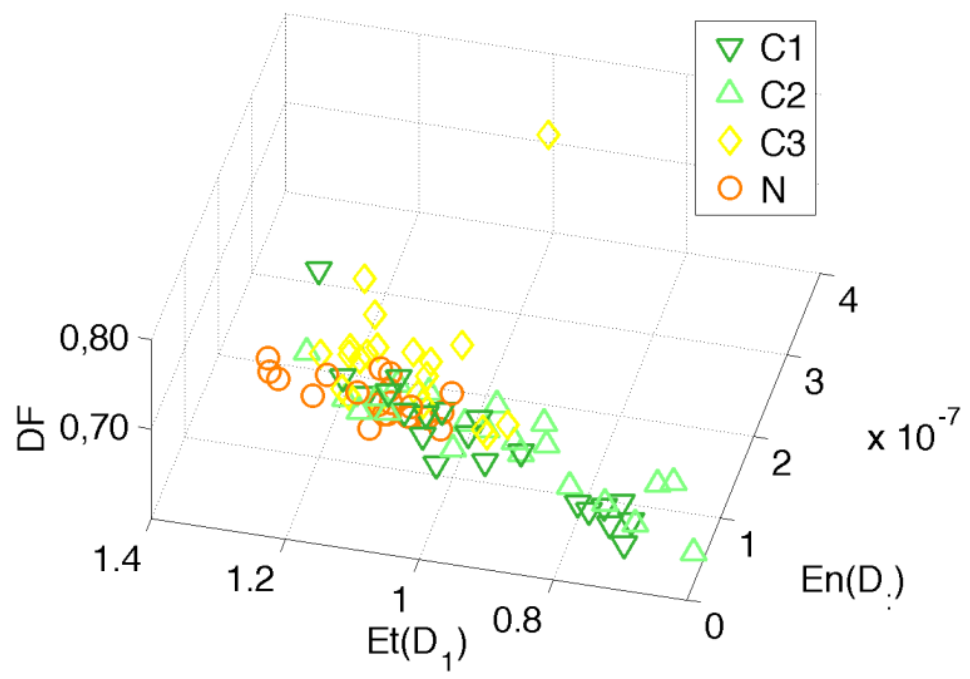

Figura 3 - Gráfico de dispersão dos valores médios de En, Et e DF, para as condições de operação C1, C2, C3 e N.

\subsection{Detecção de falhas e validação dos resultados}

Para avaliar a capacidade de discriminação dos atributos e de detecção das falhas, foi utilizada uma rede neural treinada com o algoritmo ELM. O desempenho da rede foi avaliado utilizando validação cruzada. O desempenho médio do classificador foi obtido após 100 repetições e em cada uma delas foram selecionadas aleatoriamente $80 \%$ dos vetores de atributos para a etapa de treinamento e o complemento foi utilizado para a etapa teste. 
$\mathrm{Na}$ etapa de treinamento da ELM, foram utilizados apenas dados do ensaio de alta impedância. Com o objetivo de aproximar as condições reais de operação, nas etapas de teste de validação do classificador foram acrescentados os dados de baixa impedância e removidas as condições em que o motor é acionado sem carga mecânica.

Uma matriz de confusão foi calculada para cada treinamento-teste realizado. Diferentes quantidades de neurônios na camada escondida $(\mathrm{H})$ foram avaliados. Também foram avaliadas diferentes situações de monitoramento, considerando grupos distintos de falhas. As matrizes de confusão, apresentadas nas Tabelas 2 a 5, registram o resultado correspondente ao valor médio da acurácia das 100 realizações treinamento-teste. Os resultados mostram que a falha C3, com acurácia de $84 \%$, é melhor identificada se comparada com as falhas $\mathrm{C} 1$ e C2, com as quais foram registradas acurácias de $69 \%$ e $78 \%$, respectivamente. O número de neurônios $(\mathrm{H})$ utilizados na camada escondida da ELM, para os resultados registrados nas Tabelas 2, 3 e 4, variou de 7 a 12, em função do conjunto de dados avaliado. A fim encontrar esse número ótimo de neurônios foi realizada uma busca exaustiva na faixa de 1 a 40 neurônios para cada experimento.

Tabela 2 - Matriz de confusão da acurácia média de $69 \%$ com $\mathrm{H}=8$.

\begin{tabular}{c|c|c}
\hline \multirow{2}{*}{} & \multicolumn{2}{|c}{ Predição } \\
\cline { 2 - 3 } & $\mathrm{C} 1$ & $\mathrm{~N}$ \\
\hline $\mathrm{C} 1$ & $31 \%$ & $19 \%$ \\
\hline $\mathrm{N}$ & $12 \%$ & $38 \%$ \\
\hline
\end{tabular}

Tabela 3 - Matriz de confusão da acurácia média de 78\% com H=7.

\begin{tabular}{c|c|c}
\hline \multirow{2}{*}{} & \multicolumn{2}{|c}{ Predição } \\
\cline { 2 - 3 } & $\mathrm{C} 2$ & $\mathrm{~N}$ \\
\hline $\mathrm{C} 2$ & $34 \%$ & $16 \%$ \\
\hline $\mathrm{N}$ & $6 \%$ & $44 \%$ \\
\hline
\end{tabular}

Tabela 4 - Matriz de confusão da acurácia média de 84\% com H=12.

\begin{tabular}{c|c|c}
\hline \multirow{2}{*}{} & \multicolumn{2}{|c}{ Predição } \\
\cline { 2 - 3 } & $\mathrm{C} 3$ & $\mathrm{~N}$ \\
\hline $\mathrm{C} 3$ & $41 \%$ & $9 \%$ \\
\hline $\mathrm{N}$ & $7 \%$ & $43 \%$ \\
\hline
\end{tabular}

A taxa de falsos positivos determina a proporção de falsas detecções de falhas, o que levaria a uma interrupção desnecessária da operação do motor. Para a falha $\mathrm{C} 1$, essa taxa é de $12 \%$ e diminui para a metade com a falha $\mathrm{C} 2$. Vale notar que, à medida que a falha evolui, a taxa de falsos positivos se torna menor, o que é um resultado esperado.

Embora o desempenho de classificação para as falhas C1 e C2 sejam diferentes, ambas apresentam padrões similares entre si. Isto se deve ao fato de que elas apresentam um reduzido percentual de espiras em curto circuito (menor do que 5\%). A Tabela 5 registra o resultado de um experimento no qual os conjuntos $\mathrm{C} 1$ e $\mathrm{C} 2$ representam uma única classe de falhas prematuras (sendo representadas pela classe $\mathrm{C} 1+\mathrm{C} 2$ ) e C3 representa uma classe de falhas mais severas. 
Observa-se que o desempenho é similar ao obtido apenas para as falhas C3, contudo proporcionando uma gradação na identificação da severidade da falha.

Tabela 5 - Matriz de confusão da acurácia média de 74\% com H=37.

\begin{tabular}{c|c|c|c}
\hline & \multicolumn{3}{|c}{ Predição } \\
\cline { 2 - 4 } & $\mathrm{C} 1+\mathrm{C} 2$ & $\mathrm{C} 3$ & $\mathrm{~N}$ \\
\hline $\mathrm{C} 1+\mathrm{C} 2$ & $40 \%$ & $2 \%$ & $8 \%$ \\
\hline $\mathrm{C} 3$ & $3 \%$ & $19 \%$ & $4 \%$ \\
\hline $\mathrm{N}$ & $8 \%$ & $2 \%$ & $15 \%$ \\
\hline
\end{tabular}

Observa-se que a taxa de falsos positivos registrada nos experimentos é maior nos casos em que o conjunto de dados da falha $\mathrm{C} 1$ é incluído na análise. Isto ocorre porque se tratar de uma falha que representa apenas $1,4 \%$ do número de espiras, não comprometendo significativamente o desempenho do motor. Portanto, o padrão de vibração da falhas C1 tem o mesmo comportamento daquele registrado na condição normal, o que torna difícil identificá-lo.

\section{CONCLUSÕES}

Neste trabalho foi apresentada uma metodologia para monitoramento e detecção de falhas em motores elétricos através da classificação de padrões extraídos de sinais de vibração obtidos com acelerômetros MEMS. A metodologia proposta baseia-se na análise de complexidade do sinal de vibração por meio da extração de características e encontra aplicação no monitoramento da condição de máquina assim como na identificação das falhas em seu estágio inicial.

A taxa de falsos positivos registrados nos experimentos não compromete a aplicabilidade da metodologia proposta. Isto mostra que o problema abordado não é trivial e remete a uma investigação no sentido do aumento da confiabilidade da detecção da falha.

Para trabalhos futuros pretende-se investigar as características de vibração das falhas elétricas simétricas e assimétricas, assim como as falhas mecânicas combinadas e a identificação de falhas em motores quando submetidos aos carregamentos dinâmicos.

\section{AGRADECIMENTOS}

Este trabalho recebeu suporte financeiro do programa PROAPP/IFCE 02-2012. A parte experimental recebeu suporte financeiro da FUNCAP e CAPES.

\section{REFERÊNCIAS}

1. Chakraborty, K., De, A. and Chakrabarti, A. Voltage stability assessment in power network using self organizing feature map and radial basis function, Computers and Electrical Engineering 38: 819-826, 2012.

2. Chen, Y., Ni, J.-Q., Diehl, C. a., Heber, A. J., Bogan, B. W. and Chai, L.-L. Large Scale Application of Vibration Sensors for Fan Monitoring at Commercial Layer Hen Houses, Sensors 10(12): 11590-11604, 2010. 
3. Oliveira, A. G., Bessa, R., Coelho, D. N., Medeiros, C. M. de Sá, Pontes, R. S. T. Técnicas computacionais para deteção de falhas por curto-circuito entre espiras de um motor de indução acionado por conversor de frequência. SBAI 2013 (1), 2013.

4. Falconer, K. Fractal Geometry: Mathematical Foundations and Applications, 2a. ed., John Wiley \& Sons, 2003.

5. Hassan, L. H, Moghavvemi, M., Almurib, H. A. F., Steinmayer, O. Current state of neural networks applications in power system monitoring and control. Electrical Power and Energy Systems 51: 134-144, 2013.

6. Huang, G.-B., Zhu, Q.-Y. and Siew, C.-K. (2004). Extreme learning machine: A new learning scheme of feedforward neural networks, IEEE International Joint Conference on Neural Networks. Proceedings 2: 985-990, 2004.

7. Huang, G., Zhu, Q. and Siew, C. Extreme learning machine: Theory and applications, Neurocomputing 70(1-3): 489-501, 2006.

8. Jiang, D. and Liu, C. Machine condition classification using deterioration ma- chine condition classification using deterioration feature extraction and anomaly deter- mination, IEEE Transactions on Reliability 60(1): 41-48, 2011.

9. Konar, P. and Chattopadhyay, P. Bearing fault detection of induction motor using wavelet and Support Vector Machines (SVMs), Applied Soft Computing 11(6): 4203-4211, 2011.

10. Li, B., Zhang, P.-L., Tian, H., Mi, S.-S., Liu, D.-s. and Ren, G.-q. (2011). A new feature extraction and selection scheme for hybrid fault diagnosis of gearbox. Expert Systems with Applications 38(8): 10000-10009, 2011.

11. Li, H., Zhang, Y. and Zheng, H. Application of hermitian wavelet to crack fault detection in gearbox. Mechanical Systems and Signal Processing 25(4): 1353-1363, 2010.

12. Mallat, S. A wavelet tour of signal processing, 2 ed., Academic Press, 1999.

13. Maruthi, G. S. and Vittal, K. P. Electrical Fault Detection in Three Phase Squirrel Cage Induction Motor by Vibration Analysis using MEMS Accelerometer, 2005 Interna- tional Conference on Power Electronics and Drives Systems 2: 838-843, 2005.

14. Nepomuceno, L. X. Técnicas de Manutenção Preditiva, 1a. ed., Edgard Blucher, São Paulo, 1989.

15. Paiva, H. M., Galvão, R. K. H. and Rodrigues, L. A wavelet-based multivariable approach for fault detection in dynamic systems, Revista Controle \& Automação 20(4): 455- 464, 2009.

16. Ramalho, G. L. B., Pedrosa, P., Schmidlin Jr, C. R. and Dias, S. V. Deteção de condição de máquina utilizando acelerômetro MEMS rede SOFM, SBAI 2013 (1), 2013.

17. Ramalho, G. L. B., Schmidlin Jr, C. R., Bezerra, L. D. S. and Dias, S. V. Monitoramento de condição de máquina utilizando acelerômetro MEMS, Revista Tecnologia (UNIFOR) 33(1), 2012.

18. Wang, Y., Cao, F. and Yuan, Y. A study on effectiveness of extreme learning machine, Neurocomputing 74(16): 2483-2490, 2011. 